Expert Rev Cardiovasc Ther. 2018 November ; 16(11): 815-823. doi:10.1080/14779072.2018.1534069.

\title{
Optimal medical treatment of hypertension in patients with coronary artery disease
}

\author{
Dhruv Mahtta ${ }^{1}$, Islam Y. Elgendy ${ }^{2}$, and Carl J. Pepine ${ }^{2}$ \\ 1.Department of Medicine, University of Florida, Gainesville, Florida, USA \\ 2.Department of Medicine, Division of Cardiovascular Medicine, University of Florida, Gainesville, \\ Florida, USA
}

\begin{abstract}
Introduction: Coronary artery disease (CAD) remains the leading cause of mortality and morbidity worldwide, and hypertension is its most prevalent modifiable risk factor. Patients with $\mathrm{CAD}$ and concomitant hypertension are a special population with distinct physiologic and structural alterations. Optimal blood pressure (BP) control in this population has been linked with reduction in adverse outcomes, however, excessive lowering of BP could jeopardize myocardial and cerebral perfusion.
\end{abstract}

Areas covered: Authors highlight the prevalence of the CAD and hypertension dyad, as well as the implications of various structural and physiological changes in this population. Subsequently, available data on optimal BP targets in such patients, and lastly provide more insight into the Jcurve phenomenon as well as antihypertensive agent use is discussed.

Expert commentary: Current guideline recommendations are based on data from trials such as SPRINT which did not specifically focus on the CAD population. Based on data from observational studies and post hoc analyses, the best therapeutic systolic (SBP) and diastolic (DBP) targets may be $\sim 130 \mathrm{mmHg}$ and $\sim 80 \mathrm{mmHg}$, respectively. Caution should be taken to not lower SBP below $120 \mathrm{mmHg}$ and DBP below $60 \mathrm{mmHg}$.

\section{Keywords}

Antihypertensives; Coronary auto-regulation; Endothelial dysfunction; J-curve phenomenon; Major adverse cardiovascular events; Pulse-wave velocity; Target blood pressure

\subsection{Introduction}

Coronary artery disease (CAD) is the leading cause of death in United States (US) and accounts for approximately one third of all deaths worldwide [1-2]. The dyad of CAD and hypertension is considered the most prevalent disease dyad in the older US population [3]. Hypertension remains the most prevalent modifiable risk factor for the development and

\footnotetext{
Corresponding author: Carl J. Pepine, Department of Medicine, Division of Cardiovascular Medicine, University of Florida, Gainesville, Florida Gainesville, Florida, USA, Tel.: +1 352273 9082, Fax: + 1352392 3606, carl.pepine @ medicine.ufl.edu. Reviewer Disclosures

Peer reviewers on this manuscript have no relevant financial relationships or otherwise to disclose.
} 
progression of this disease process in US patients. The association between uncontrolled hypertension and increased incidence of major adverse cardiovascular events is established [4-5]. Several studies have shown significant reductions in cardiovascular mortality with improved blood pressure control [6-9]. This relationship between adverse clinical outcomes and hypertension is amplified in patients with underlying CAD [10]. The existence of a Jcurve phenomenon was proposed by findings suggestive of worsened ischemia-related outcomes with the extremes of blood pressure (BP) [11-14]. This observation has been attributed to the interaction between lower BP and specific structural and physiological changes that may exist in patients with CAD. The 2017 American College of Cardiology (ACC) and American Heart Association (AHA) guidelines have suggested a BP threshold of $\geq 130 / 80 \mathrm{mmHg}$ for initiation of antihypertension drug treatment in the general adult population [15], lowered from the 140/90 $\mathrm{mmHg}$ threshold suggested by the 2015 ACC/AHA guidelines [16]. Besides lifestyle modification, the optimal treatment of hypertensive patients with concomitant CAD continues to evolve, with special consideration toward specific pharmacological agents as well as BP targets.

\subsection{Physiological link between hypertension and coronary artery disease}

Many of the physiological mechanisms involved in pathogenesis of hypertension also play a key role in the development of atherosclerosis in the epicardial coronary vessels as well as dysfunction in the microvessels. Increased sympathetic drive centrally via activation of the renin-angiotensin-aldosterone system (RAAS), increased oxidative stress and inflammatory cytokines, endothelial and microvascular dysfunction, and deficiency in vasodilators such as nitric oxide and prostacyclin are among the many contributing factors. Endothelial dysfunction remains most pronounced in patients with vascular diseases such as CAD and hypertension. This includes an imbalance between bioavailable vasodilators (nitric oxide and prostaglandin E) and vasoconstrictors (endothelin and angiotensin II), as well as prothrombotic and antithrombotic mediators thereby contributing toward elevated blood pressure and athero-thrombotic risk. The increased sympathetic drive activates RAAS, increasing production of angiotensin II and aldosterone which have various downstream vasotoxic effects [16]. Furthermore, injured endothelium releases inflammatory cytokines which potentiate oxidative stress and perpetuate vascular inflammation, thereby resulting in initiation and progression of CAD, as well as microvascular disease [17].

Besides the aforementioned physiologic changes which exist in hypertensive patients, certain key physical and hemodynamic forces are also at play. Long-standing hypertension results in remodeling of coronary arteries as well as intra-myocardial structure.

Hypertension, specifically elevations in the systolic blood pressure (SBP), results in an increased impedance to left ventricular output which thereby increases left ventricular (LV) wall tension. Both of these factors eventually increase myocardial wall thickness or LV hypertrophy thereby leading to increased cardiac metabolic and oxygen demands. The hypertrophy also contributes to disordered LV relaxation with also influences the hydraulic determinant of coronary blood flow (e.g. diastolic-pressure time).

Along with aging, hypertension also results in thinning of vascular elastin and deposition of collagen in the vasculature. This results in arterial stiffening and loss of its innate elasticity. 
The loss of arterial elasticity in the coronary vasculature hinders the auto-regulatory process of the cardiac circulation, which serves as an important mechanism to preserve coronary perfusion in patients with CAD [18]. Animal studies have shown that left ventricular hypertrophy as well as occlusive CAD limit the auto-regulation mechanisms of the coronary vasculature $[19,20]$. Due to this inability to auto-regulate, the coronary vasculature is unable to dilate beyond a certain point to maintain adequate coronary perfusion. The same loss of arterial elasticity which exists in the coronaries also takes place in the aortic arterial tree. This results in increased pulse-wave velocity thereby causing the reflected blood pressure pulse wave to reach the aortic valve before its closure. This is in contrast with normotensive patients, in whom aortic elasticity results in a slower velocity of the pulse wave, allowing it to reach the aortic valve after its closure. This results in higher diastolic blood pressure (DBP) at the entrance to the aorta, which increases LV systolic wall tension and myocardial oxygen demand. However, while elevated aortic diastolic pressure from the addition of the reflected pressure waves may augment coronary perfusion [21], reflected blood flow waves subtract from the forward flow (e.g. cardiac output). Thus, energy is "wasted" overcoming these hydraulic forces.

Studies have documented improvement in cardiovascular outcomes with treatment of hypertension in patients with CAD. This is attributed to lowering the SBP, as this is the culprit in increased left ventricular outflow impedance, resulting hypertrophy, and increased myocardial oxygen demand. With regard to lowering the DBP, debate continues regarding the safety threshold. Myocardial perfusion occurs primarily during diastole. Given what we know about increased myocardial demand in hypertensive patients, increased pulse-wave velocity, and decreased auto-regulatory mechanisms in hypertensive patients with $\mathrm{CAD}$, it is expected that excessive lowering of DBP, at some critical pressure, would impair perfusion in these patients. However, it is important to note that data regarding the lower limit of coronary auto-regulation exist only from animal studies, and data from human coronary circulation are lacking. The notion of lowering DBP below the lower limit of auto-regulation in the coronary vasculature has given rise to the J-curve phenomenon; which suggests that an increased risk of coronary events occurs at both ends of the spectrum. Unfortunately, robust data are lacking and currently under much debate regarding the lower limit of DBP which would correspond to the lower limit of auto-regulation in coronary vasculature and would result in increased coronary events. Moreover, patients with CAD are also likely to have concomitant atherosclerosis of the cerebral vasculature, to which blood flow may be compromised with excessive lowering of DBP without proper auto-regulatory mechanisms in place [22].

\subsection{Prevalence and implications of hypertension in patients with coronary artery disease}

In a large analysis highlighting the prevalence of various risk factors, including hypertension, in patients with CAD [23], over 122,000 patients presenting with ST-elevation myocardial infarction (STEMI), non-ST-elevation myocardial infarction (NSTEMI), unstable angina, or undergoing percutaneous coronary intervention (PCI) were analyzed from 14 international randomized trials. The authors found that in patients with pre-existing 
$\mathrm{CAD}$, the prevalence of hypertension ranged from $30 \%$ to $70 \%$. As expected, hypertension was more prevalent among these women with CAD as well as elderly patients with CAD. The true prevalence of hypertension in patients with CAD may be higher than what authors found in this study. The reason for this is twofold: first, almost one third of the patients with hypertension are unware that they are hypertensive [24], and second, patients with stable ischemic heart disease were missing from the study population since only patients with STEMI, NSTEMI, unstable angina, and those undergoing PCI (presumably with accelerated ischemia-related symptoms) were included. With the new 2017 ACC/AHA definition of hypertension, the prevalence is expected to increase by $13.7 \%$ making the total prevalence of hypertension to be $45.6 \%$ of all American adult population [25].

Uncontrolled hypertension in patients with known CAD has been the topic of multiple investigations. A retrospective analysis of the Valsartan Antihypertensive Long-term Use Evaluation (VALUE) trial [26] evaluated cardiovascular outcomes in patients with CAD and hypertension. Patients were divided into three subgroups: those with controlled hypertension (defined as BP <140/90) with one agent, those with controlled hypertension with two agents, and those who remained uncontrolled with two agents. The primary outcome was the first occurrence of cardiovascular death, nonfatal myocardial infarction, or stroke. The authors demonstrated that patients in the group where hypertension was controlled with two agents had significantly higher rates of the primary outcome compared with those whose hypertension was controlled with only one agent $(11.1 \%$ vs. $8.5 \%$, p $<0.05)$. Patients with uncontrolled hypertension had a non-significant increase in adverse cardiovascular events compared with patients controlled on two agents. However, it is important to note that the confounding effect of choice of medications used to reduce BP was not evaluated by this trial and this may be at play when it comes to such findings as reported by the authors. The findings from this study were supported by another trial in patients with CAD [27] which showed that compared with patients with CAD and controlled hypertension, those with resistant or uncontrolled hypertension encountered higher rates of adverse outcomes, including all-cause death and nonfatal myocardial infarction (hazard ratio [HR] 1.27, 95\% confidence interval [CI] 1.13-1.43).

In a retrospective analysis of women age $\leq 45$ years undergoing PCI, among other cardiovascular risk factors, existence of hypertension was an independent predictor of major adverse cardiovascular outcomes at 36-month follow up (HR 3.07, 95\% CI 1.30-7.24, $\mathrm{p}=0.01)[28]$.

\subsection{Clinical evidence regarding target levels of treatment}

Having established that uncontrolled hypertension, on the background of CAD, results in increased adverse cardiovascular events, investigators subsequently focused on whether lower is better was true in terms of BP. A multitude of randomized trials have evaluated intensive versus standard control of BP in patients with risk factors for cardiovascular disease such as diabetes. An earlier randomized trial conducted by the United Kingdom Prospective Diabetes Study Group evaluated rates of macrovascular and microvascular complications in diabetic patients with tight versus less tight BP control [29]. The results showed that tight BP control (mean BP of $144 / 82 \mathrm{mmHg}$ ) was associated with less

Expert Rev Cardiovasc Ther. Author manuscript; available in PMC 2019 November 01. 
microvascular and macrovascular complications as compared with less tight BP control (mean BP of $154 / 87 \mathrm{mmHg}$ ). This trial was followed by a decade of additional investigation as summarized by Xie et al in a meta-analysis [30]. Based on their analysis of over 44,000 patients who were followed over a mean of 3.8 years, the authors concluded that an intensive $\mathrm{BP}$ control regimen (mean BP of $133 / 76 \mathrm{mmHg}$ ) provided more vascular protection as compared with "then standard" BP regimens (mean BP of 140/81 mmHg).

The Action to Control Cardiovascular Risk in Diabetes (ACCORD) trial evaluated cardiovascular outcomes with additional lowering of SBP [31]. The investigators randomized 4,733 diabetic patients from US and Canada into either a standard SBP lowering $(<140 \mathrm{mmHg}$ ) or an intensive SBP lowering (i.e., $<120 \mathrm{mmHg}$ ) strategy. At mean 4.7 years mean, there were no statistically significant differences in the primary outcome of nonfatal myocardial infarction, nonfatal stroke, or death from cardiovascular causes comparing the intensive therapy vs. the standard therapy arm (HR 0.88, 95\% CI $0.73-1.06 ; \mathrm{P}=0.20$ ). On the other hand, the risk of adverse outcomes associated with anti-hypertensive therapy was higher in the intensive therapy cohort $(3.3 \%$ vs. $1.3 \% ; \mathrm{P}<0.001)$. It is important to note that only 1,593 out of 4,733 patients ( 34\%) enrolled in this trial had known CAD. In the subgroup analysis of patients with CAD, intensive SBP lowering was not associated with a lower rate of the primary outcome as compared to a standard SBP lowering strategy (2.98\% vs. $3.43 \%$, p=0.78). Importantly, a subsequent post-hoc, non prespecified exploratory analysis of ACCORD showed that after correcting for differences in glycemic regimens, patients in the intensive SBP control group had reduction in stroke risk compared with those receiving standard therapy [32]. Comparing the results of the pre-specified analysis from ACCORD with the pre-ACCORD trials, the discrepancies in the findings may be attributed to lack of control for glycemic index and comparison of intensive regimen with standard regimen vs. less intense regimen, which in some cases resulted in patient remaining hypertensive.

The Systolic Blood Pressure Intervention Trial (SPRINT) [33] enrolled over 9,000 patients with risk factors or at high risk of CVD randomized into either intensive treatment with a target SBP $<120 \mathrm{mmHg}$ or standard treatment with a target SBP $<140 \mathrm{mmHg}$. Only 1,562 patients (16.7\%) enrolled in SPRINT had known clinical cardiovascular disease. Patients with a history of stroke or diabetes were excluded from this trial unlike patients who were enrolled in the ACCORD trial. The primary outcome was a first occurrence of myocardial infarction, stroke, acute coronary syndromes, heart failure, or cardiovascular death. This trial was prematurely terminated because of a significantly lower rate of primary outcome, and also mortality, in the intensive treatment group. At a median follow up period of only 3.26 years, patients in the intensive treatment group had a lower incidence of primary outcome (HR 0.75, 95\% CI $0.64-0.89 ; \mathrm{P}<0.001$ ) and all-cause mortality (HR 0.73, CI $0.60-0.90$; $\mathrm{p}=0.003$ ) compared with those in the standard treatment group. The incidence of adverse events such as hypotension, syncope, electrolyte derangements, or acute kidney injury was significantly higher in those assigned intensive compared with standard treatment. Despite this increased risk of adverse events in the intensive treatment group, patient-reported outcomes were similar in both groups [34]. Of note, in both ACCORD and SPRINT only a small minority of patients had known CAD. Hence, there is an important knowledge gap related to how to best treat hypertensive patients with CAD. 
Lastly, the main concerns regarding the existence of the $\mathrm{J}$ curve and lowering the DBP to an optimal threshold were left unaddressed at the conclusion of most of these randomized trials. Thus, the data regarding the best DBP target in hypertensive patients with CAD have been extrapolated mainly from retrospective studies and post hoc analyses of older randomized trials (Table 1).

The Valsartan Antihypertensive Long-term Use Evaluation (VALUE) was a randomized trial of 15,244 hypertensive patients, about half of whom had CAD [35]. At a mean follow up of 4.2 years, although there were no significant differences in the primary cardiovascular outcome with regard to use of valsartan versus amlodipine; there were notable differences regarding BP in these patients. Patients with DBP $\geq 90 \mathrm{mmHg}$ had an increased incidence of $\mathrm{CV}$ events compared with those with DBP $<90 \mathrm{mmHg}$. However, no differences were found in primary outcome between patients with DBP $<70 \mathrm{mmHg}$ versus those with $\geq 70 \mathrm{mmHg}$, findings that did not support the J-curve hypothesis. Patients with SBP $\geq 150 \mathrm{mmHg}$ experienced significantly increased rates of cardiovascular events compared with patients with SBP $<130 \mathrm{mmHg}$ and those with SBP 130-149 mmHg. Another key finding was that the lower DBP threshold for myocardial infarction was $76 \mathrm{mmHg}$ and for stroke was 60 mmHg. With lower DBP, the ratio of myocardial infarctions to strokes was increased. This may suggest the vulnerability of different organs at different DBP thresholds.

The International Verapamil-trandolapril Study (INVEST) was a large, international, multicenter trial, with prospective randomized blinded endpoint (PROBE) design [36]. That only patients with prior diagnosis of CAD were enrolled was a unique aspect of this trial, in contrast to all other prior studies where only a fraction of patients had CAD or subjects were only at-risk for cardiovascular diseases. Over 22,000 patients were enrolled, and the primary outcome was the first occurrence of all-cause mortality, nonfatal myocardial infarction, or nonfatal stroke. Given that older patients ( $>60$ years of age) have a high prevalence of CAD, Bangalore et al conducted a secondary analysis from the INVEST [37] and found that in hypertensive patients aged $>60$ years with known CAD, a target SBP of $<140 \mathrm{mmHg}$ provided more benefit in terms of cardiovascular mortality, myocardial infarction, and allcause mortality compared with a target SBP either $140-150 \mathrm{mmHg}$ or $>150 \mathrm{mmHg}$.

Compared with the reference group of $<140 \mathrm{mmHg}$, SBP between 140 and $<150 \mathrm{mmHg}$ was associated with an increased risk of cardiovascular mortality (HR: $1.34,95 \% \mathrm{CI}$ : 1.01-1.77, $\mathrm{p}=0.04$ ), total stroke (HR: 1.89, 95\% CI: 1.26-2.82, $\mathrm{p}=0.002$ ), and nonfatal stroke (HR: 1.70, 95\% CI: 1.06-2.72, $\mathrm{p}=0.03$ ). In the long term (median 11.6 years) follow up of the US cohort (17,000 patients) of the INVEST, achieving a SBP 130 to $140 \mathrm{mmHg}$ was associated with lower all-cause mortality [10]. It is important to note that data regarding BP control beyond the active follow up of the trial were not available in this later analysis from the US cohort. Thus, these data, together with newly published ASCOT data [38], support the notion that achieving excellent BP control for a period of at least 2 years has a "legacytype effect" to reduce mortality over the long term (>11 years) in hypertensive patients with CAD.

Few studies have examined the effect of lowering the DBP in hypertensive patients with CAD. The association between lowering DBP and myocardial damage, measured by cardiac troponin-T (cTnT), was analyzed in the Atherosclerosis Risk In Communities (ARIC) cohort 
[39]. Patients with DBP $80-89 \mathrm{mmHg}$ were compared with those with DBP $<60 \mathrm{mmHg}$ and those with DBP 60-69 mmHg. The results showed that achieving low DBP (particularly $<60$ $\mathrm{mmHg}$ ) in hypertensive patients was associated with increased risk of events related to coronary heart disease and subclinical myocardial damage. The take-away point from this analysis was that care should be exercised when titrating SBP below $140 \mathrm{mmHg}$ (to the 120 mmHg goal as suggested by SPRINT), and DBP should not be allowed to fall below 60-70 mmHg. In another analysis of the US cohort of INVEST [40] which focused on the implications of lowering DBP along with SBP, patients were divided on the basis of SBP: $<120,120-<130,130-<140$, and $\geq 140 \mathrm{mmHg}$. Subsequently, patients in each SBP category were subdivided into tertiles of DBP: low, middle, or high. This analysis demonstrated that risk of long-term mortality (median 11.6 years) with excessive lowering of DBP becomes more prominent with concomitant intensive lowering of SBP to below $120 \mathrm{mmHg}$. These conclusions were in line with an analysis of $\sim 22,000$ hypertensive patients with CAD from the CLARIFY registry [41], which showed that SBP $<120 \mathrm{mmHg}$ was associated with an increased risk of cardiovascular death, myocardial infarction, and stroke, as well as DBP $<70 \mathrm{mmHg}$. The risk of adverse outcomes was lower with a SBP $\sim 130 \mathrm{mmHg}$. Although some studies had analyzed thresholds for SBP and DBP independently, there is paucity of data regarding the interactions between concomitant lowering of SBP and DBP. For this reason, an analysis from a large registry evaluated the prognostic effects of pulse pressure (PP) and found an association between widening PP and increased adverse cardiovascular events [42]. However, these findings were offset by large analysis from the INVEST cohort which only showed a weak association between PP and adverse cardiovascular outcomes [43].

In summary, data regarding SBP and DBP control in hypertensive patients with CAD are driven from post-hoc analyses of randomized controlled trials which were not designed to compare different BP thresholds, as well as from observational studies. Additionally, whether concomitant lowering of SBP and DBP truly affects mortality or is simply a surrogate for poor overall health in these subjects is a question requiring additional investigation. Nonetheless, it appears that aggressive lowering of SBP below $120 \mathrm{mmHg}$ may be linked with a worse prognosis, whereas adopting a more conservative strategy of SBP 120-140 mmHg in older adults with CAD may be associated with improved outcomes as compared with SBP >140 mmHg. Additionally, with SBP 120-140 mmHg, there is no increase in mortality even at lower DBP.

\subsection{Optimal drug choice}

Current practice suggests use of a multidrug regimen, at lower doses, as opposed to a single drug at maximally tolerated dose for management of hypertension. This is in part due to the complementary effects of several drugs acting on different pathways and also due to avoidance of side effects of a particular drug when titrated to its maximally tolerated dose and then reducing the dose to limit the side effect. Although beta blockers remained a standard of care for hypertension treatment in the $20^{\text {th }}$ century, their sub-par antihypertensive effects in older patients have been well documented by several studies [44]. Hence, recommendations for their use has been curtailed except, in patients with recent myocardial infarction, left ventricular systolic dysfunction, and/or clinical heart failure 
[45-47]. However, since hypertensive patients with co-existing CAD often have extensive vascular disease with associated conduit vessel stiffening, the heart rate slowing effect of beta blockers augments the functional effects of conduit vessel stiffening [48]. This has the potential to increase systolic blood pressure and limit the benefit of beta blockers. Hence, in current practice, beta blocker use is limited to blood pressure control among younger patients and for older patients only if they have acute MI, systolic heart failure or angina.

The safety and efficacy of thiazide and thiazide-like diuretics, angiotensin-converting enzyme inhibitors (ACE-I), angiotensin receptor blockers (ARB), and calcium channel blockers have been assessed by multiple trials [49-57]. However, most of these hypertension trials were conducted in populations with a low prevalence of co-existing CAD, thereby making it difficult to reach conclusions.

In hypertensive patients with symptomatic chronic $\mathrm{CAD}$, beta-blockers and calcium channel blockers assert well-established antianginal/anti-ischemic effects and in addition, contribute to optimization of BP among younger patients. These two drug classes are often chosen over other agents (such as ACE-I, ARB, or diuretics) which do not provide additional antianginal benefit. A comparison of beta-blockers and non-dihydropyridine calcium channel blockers specifically in patients with hypertension and co-existing CAD showed no significant differences in terms of death, nonfatal myocardial infarction, or nonfatal strokes between the two drug classes [36]. In the light of the results from INVEST and other large randomized trials, no specific recommendations exist regarding the choice of anti-hypertensive agents for patients with CAD. However, the choice of anti-hypertensives should be tailored toward an individual patients' symptoms (such as exertional angina) or co-morbid conditions (heart failure, diabetes, chronic kidney disease, left ventricular dysfunction, or valvulopathies).

\subsection{Expert commentary}

The prevalence of CAD in patients with hypertension poses a special challenge during treatment of BP. Over the last two decades, several studies have suggested a J-curve phenomenon and advised caution while lowering BP in these patients with coronary atherosclerosis. The reason for this caution is due to the changes in coronary autoregulation, increased pulse wave velocity, and concomitant cerebral atherosclerosis as well as the potential for microvascular dysfunction in the coronary, cerebral and renal circulations. The data from earlier studies pose a discrepancy regarding the J-curve phenomenon and safety threshold for lowering BP in patients with CAD. These discrepancies have been reflected in the different guideline recommendations (Table 2) [15,16,58,59]. For example, the members of the JNC-8 panel, which did not provide a specific endorsement for the CAD population, recommended a SBP threshold of $<150 \mathrm{mmHg}$ in those $>60$ years, and $<140 \mathrm{mmHg}$ in those $<60$ years of age. The 2015 ACC/AHA guidelines for hypertension control in the CAD population recommended a BP target $<140 \mathrm{mmHg}$. Those recommendations have now been updated by the $2017 \mathrm{AHA} / \mathrm{ACC}$ guidelines for hypertension management, where a BP $<130 / 80 \mathrm{mmHg}$ recommended in this population. A similar recommendation has been provided by the updated 2018 ESC guidelines for the management of hypertension, except in those aged $\searrow 65$ years, in which a SBP target of 130-140 $\mathrm{mmHg}$ is recommended [60]. These recommendations were based mainly on the data from the SPRINT trial, which did 
not specifically focus on the CAD population and actually had relatively few patients with documented CAD.

Moreover, there have been concerns regarding the "unobserved" SBP measurement in the SPRINT, with some suggestions that this unobserved measurement could have resulted in a 10-15 mmHg lower level [61]. To date there has been no randomized trial which compared the different therapeutic SBP targets in hypertensive patients with CAD, and the evidence has been driven from registries or post-hoc analyses of randomized trials. Based on the results of the available observational studies, it might be plausible that the best therapeutic SBP target in these patients is $\sim 130 \mathrm{mmHg}$, but physicians should be cautious in not reducing the SBP below $120 \mathrm{mmHg}$. Similarly, a DBP $<80 \mathrm{mmHg}$ appears to be an adequate target with caution not to lower the DBP $<70 \mathrm{mmHg}$, and specifically not $<60 \mathrm{mmHg}$. There are no recommendations regarding any specific anti-hypertensive agent, the choice of which should be tailored according to the patient's symptoms, and consideration for those with an anti-angina effect (e.g., calcium channel blockers and beta blockers) for those with uncontrolled angina symptoms. Furthermore, given recent data and ongoing trials on renal artery denervation for uncontrolled and treatment resistant hypertension, it would be interesting to obtain outcomes data from well-designed trials evaluating the effects of renal artery denervation versus standard medical therapy in hypertensive patients with CAD. Finally, there should be careful emphasis on secondary preventive measures in these patients such as smoking cessation, diabetes control, weight loss, and regular exercise (Figure 1).

\title{
7.0 Five-year view
}

Continuous efforts to promote primary prevention by reducing sodium consumption, and increasing physical activity, and through the emphasis on reducing the burden of risk factors such as smoking cessation, and cholesterol management [62], it is expected that the prevalence of the dyad of hypertension and CAD might decrease in the upcoming years. Further observational data are expected to further explore the best therapeutic SBP target among this high-risk cohort. It is unlikely that a randomized trial will be conducted to compare the therapeutic BP targets in this population. With the emergence of renal denervation therapy, it is anticipated to see studies that would focus on the potential benefit (i.e., BP control, reduction in the number of anti-hypertensive agents) among hypertensive patients with CAD.

\section{Acknowledgments}

\author{
Funding \\ This paper was not funded. \\ Declaration of interest \\ CJ Pepine receives support from the Gatorade Trust through funds distributed by the University of Florida, \\ Department of Medicine; NIH NCATS—University of Florida Clinical and Translational Science UL1TR001427; \\ and PCORnet-OneFlorida Clinical Research Consortium CDRN-1501-26692. CJ Pepine also reports grant support \\ (significant) from Adelphi Values (Qualitative MVA), Amorcyte (PRESERVE), Athersys (MI-NSTEMI), BioCardia \\ (CardiAMP), Brigham and Women's Hospital (INVESTED), Capricor (ALLSTAR), Cytori Therapeutics \\ (ATHENA), Duke Univ. (ADAPTABLE), Gilead Sciences Inc. (RWISE, Univ. FL site), Merck \& Co. Inc. \\ (VICTORIA), Mesoblast (TEVA, Univ. FL site), NIH/NHLBI (CONCERT), US Dept. of Defense (WARRIOR),
}


Ventrix (CV-201); educational support (modest) for the Vascular Biology Working Group from Amgen Inc., AstraZeneca Pharmaceuticals, Boehringer Ingelheim Pharmaceuticals Inc., Daiichi Sankyo, Ionis, Relypsa; Consultant fees/honoraria (modest) from Amgen Inc., AstraZeneca Pharmaceuticals, Bayer Healthcare Pharmaceuticals, Gilead, Merck and (significant) from Ironwood Pharmaceuticals Inc. and SLACK Inc.; is a Task force member (no compensation) for Foundation for the Accreditation of Cellular Therapy. The authors have no other relevant affiliations or financial involvement with any organization or entity with a financial interest in or financial conflict with the subject matter or materials discussed in the manuscript apart from those disclosed.

\section{References}

Papers of special note have been highlighted as:

$*$ of interest

** of considerable interest

1. National Center for Health Statistics (US). Health, United States, 2015: with special feature on racial and ethnic health disparities. Hyattsville, MD: National Center for Health Statistics (US); 2016 5. Report no.: 2016-1232.

2. World Health Organization (WHO). Causes of death 2008: data sources and methods. Department of Health Statistics and Informatics, World Health Organization, Geneva 2011:1-28.

3. Benjamin EJ, Blaha MJ, Chiuve SE, et al. Heart disease and stroke statistics-2017 update: a report from the American Heart Association. Circulation. 2017;135(10):e146-603. [PubMed: 28122885]

4. Lewington S, Clarke R, Qizilbash N, et al. Age-specific relevance of usual blood pressure to vascular mortality: a meta-analysis of individual data for one million adults in 61 prospective studies. Lancet. 2002;360:1903-1913. [PubMed: 12493255]

5. Dawber TR, Moore FE, Mann GV. Coronary heart disease in the Framingham study. Am J Public Health Nations Health. 1957;47:4-24.

6. Davis BR, Cutler JA, Gordon DJ, et al. Rationale and design for the antihypertensive and lipid lowering treatment to prevent heart attack trial (ALLHAT). Am J Hypertens. 1996;9(4):342-360. [PubMed: 8722437]

7. Dorresteijn JAN, van der Graaf Y, Spiering W, et al. Relation between blood pressure and vascular events and mortality in patients with manifest vascular disease. Hypertension. 2012;59:14-21. [PubMed: 22068865]

8. Five-year findings of the hypertension detection and follow-up program. I. Reduction in mortality of persons with high blood pressure, including mild hypertension. Hypertension Detection and Followup Program Cooperative Group. JAMA. 1979;242:2562-2571. [PubMed: 490882]

9. Hansson L, Zanchetti A, Carruthers SG, et al. Effects of intensive blood pressure lowering and low dose aspirin in patients with hypertension: principal results of the Hypertension Optimal Treatment (HOT) randomized trial- HOT Study Group. Lancet. 1998;351:1755-1762. [PubMed: 9635947]

10. Elgendy IY, Bavry AA, Gong Y, et al. Long-term mortality in hypertensive patients with coronary artery disease. Hypertension. 2016;68(5):1110-1114. [PubMed: 27620390]

11. Messerli FH, Mancia G, Conti CR, et al. Dogma disputed: can aggressively lowering blood pressure in hypertensive patients with coronary artery disease be dangerous? Ann Intern Med. 2006;144(12):884-893. [PubMed: 16785477]

12. Protogerou AD, Safar ME, Iaria $P$, et al. Diastolic blood pressure and mortality in the elderly with cardiovascular disease. Hypertension. 2007;50(1):172-180. [PubMed: 17515449]

13. Cruickshank JM. Coronary flow reserve and the J curve relation between diastolic blood pressure and myocardial infarction. BMJ. 1988;297(6658):1227-1230. [PubMed: 3145062]

14. Dorresteijn JAN, van der Graaf Y, Spiering W, et al.; Secondary Manifestations of Arterial Disease Study Group. Relation between blood pressure and vascular events and mortality in patients with manifest vascular disease: J-curve revisited. Hypertension. 2012;59(1):14-21. [PubMed: 22068865]

15. Whelton PK, Carey RM, Aronow WS, et al. 2017 ACC/AHA/AAPA/ABC/ACPM/AGS/ APhA/ASH/ASPC/NMA/PCNA Guideline for the Prevention, Detection, Evaluation, and Management of High Blood Pressure in Adults: a report of the American College of Cardiology/

Expert Rev Cardiovasc Ther: Author manuscript; available in PMC 2019 November 01. 
American Heart Association Task Force on Clinical Practice Guidelines. J Am Coll Cardiol. 2018;71(19):e127-e248. [PubMed: 29146535] **This serves as the most updated ACC/AHA 2017 Hypertension Guidelines

16. Rosendorff C, Lackland DT, Allison M, et al. Treatment of hypertension in patients with coronary artery disease: a scientific statement from the American Heart Association, American College of Cardiology, and American Society of Hypertension. Hypertension. 2015;65(6):1372-1407. [PubMed: 25828847]

17. Wallace SM, McEniery CM, Mäki-Petäjä KM, et al. Isolated systolic hypertension is characterized by increased aortic stiffness and endothelial dysfunction. Hypertension. 2007;50(1):228-233. [PubMed: 17502493]

18. Casadonte L, Verhoeff BJ, Piek JJ, et al. Influence of increased heart rate and aortic pressure on resting indices of functional coronary stenosis severity. Basic Res Cardiol. 2017;112(6):61. [PubMed: 28905113]

19. Rouleau JR, Simard D, Blouin A, et al. Angiotensin inhibition and coronary autoregulation in a canine model of LV hypertrophy. Basic Res Cardiol. 2002;97(5):384-391. [PubMed: 12200638]

20. Hoffman JI. Heterogeneity of myocardial blood flow. Basic Res Cardiol. 1995;90(2):103-111. [PubMed: 7646414]

21. O'Rourke MF, Hashimoto J. Mechanical factors in arterial aging: a clinical perspective. J Am Coll Cardiol. 2007;50(1):1-3. [PubMed: 17601538]

22. Craven TE, Ryu JE, Espeland MA, et al. Evaluation of the associations between carotid artery atherosclerosis and coronary artery stenosis. A case-control study. Circulation. 1990;82(4):12301242. [PubMed: 2205416]

23. Khot UN, Khot MB, Bajzer CT, et al. Prevalence of conventional risk factors in patients with coronary heart disease. JAMA. 2003;290(7):898-904. [PubMed: 12928466]

24. Hyman DJ, Pavlik VN. Characteristics of patients with uncontrolled hypertension in the United States. N Engl J Med. 2001;345:479-486. [PubMed: 11519501]

25. Muntner P, Carey RM, Gidding S, et al. Potential US population impact of the 2017 ACC/AHA High Blood Pressure Guideline. J Am Coll Cardiol. 2018;71(2):109-118. [PubMed: 29146532]

26. Weber MA, Julius S, Kjeldsen SE, et al. Cardiovascular outcomes in hypertensive patients: comparing single-agent therapy with combination therapy. J Hypertens. 2012;30(11):2213-2222. [PubMed: 23011525]

27. Smith SM, Gong Y, Handberg E, et al. Predictors and outcomes of resistant hypertension among patients with coronary artery disease and hypertension. J Hypertens. 2014;32(3):635. [PubMed: 24299915]

28. Liu W, Mukku VK, Liu YY, et al. Long-term follow up of percutaneous coronary intervention of coronary artery disease in women $\leq 45$ years of age. Am J Cardiol. 2013;112(7):918-922. [PubMed: 23791012]

29. UK Prospective Diabetes Study Group. Tight blood pressure control and risk of macrovascular and microvascular complications in type 2 diabetes: UKPDS 38. BMJ. 1998;317(7160):703-713. [PubMed: 9732337]

30. Xie X, Atkins E, Lv J, et al. Effects of intensive blood pressure lowering on cardiovascular and renal outcomes: updated systematic review and meta-analysis. Lancet. 2016;387(10017):435-443. [PubMed: 26559744]

31. ACCORD Study Group. Effects of intensive blood-pressure control in type 2 diabetes mellitus. $\mathrm{N}$ Engl J Med. 2010;2010(362):1575-1585.*One of the landmark trials on effects of intense blood pressure control and clinical outcomes

32. Margolis KL, O'Connor PJ, Morgan TM, et al. Outcomes of combined cardiovascular risk factor management strategies in type 2 diabetes: the ACCORD randomized trial. Diabetes Care. 2014;37(6):1721-1728. [PubMed: 24595629]

33. SPRINT Research Group. A randomized trial of intensive versus standard blood-pressure control. N Engl J Med. 2015;2015(373):2103-2116.*Landmark trial evaluating clinical outcomes as a result of intense versus standard blood pressure management in non-diabetic and patients without prior strokes 
34. Berlowitz DR, Foy CG, Kazis LE, et al. Effect of intensive blood-pressure treatment on patientreported outcomes. N Engl J Med. 2017;377(8):733-744. [PubMed: 28834483]

35. Kjeldsen SE, Berge E, Bangalore S, et al. No evidence for a J-shaped curve in treated hypertensive patients with increased cardiovascular risk: The VALUE trial. Blood Press. 2016;25(2):83-92. [PubMed: 26511535]

36. Pepine CJ, Handberg EM, Cooper-DeHoff RM, et al. A calcium antagonist vs a non-calcium antagonist hypertension treatment strategy for patients with coronary artery disease: the International Verapamil-Trandolapril Study (INVEST): a randomized controlled trial. JAMA. 2003;290(21):2805-2816. [PubMed: 14657064] **INVEST Trial was the first randomized prospective trial which enrolled patients with prior known history of CAD.

37. Bangalore S, Gong Y, Cooper-DeHoff RM, et al. 2014 Eighth Joint National Committee panel recommendation for blood pressure targets revisited: results from the INVEST study. J Am Coll Cardiol. 2014;64(8):784-793. [PubMed: 25145522]

38. Gupta A, Mackay J, Whitehouse A, Godec T, Collier T, Pocock S, Poulter N, Sever P. Long-term mortality after blood pressure-lowering and lipid-lowering treatment in patients with hypertension in the Anglo-Scandinavian Cardiac Outcomes Trial (ASCOT) Legacy study: 16-year follow-up results of a randomised factorial trial. Lancet. 2018824 pii: S0140-6736(18)31776-8. Doi: 10.1016/S0140-6736(18)31776-8 [Epub ahead of print]

39. McEvoy JW, Chen Y, Rawlings A, et al. Diastolic blood pressure, subclinical myocardial damage, and cardiac events: implications for blood pressure control. J Am Coll Cardiol. 2016;68(16):17131722. [PubMed: 27590090]

40. Wokhlu A, Smith SM, Gong Y, et al. Mortality implications of lower DBP with lower achieved systolic pressures in coronary artery disease: long-term mortality results from the INternational VErapamil-trandolapril STudy US cohort. J Hypertens. 2018;36(2):419-427. [PubMed: 28938338]

41. Vidal-Petiot E, Ford I, Greenlaw N, et al.; CLARIFY Investigators. Cardiovascular event rates and mortality according to achieved systolic and diastolic blood pressure in patients with stable coronary artery disease: an international cohort study. Lancet. 2016;388(10056):2142-2152. [PubMed: 27590221]

42. Selvaraj S, Steg PG, Elbez Y, et al.; REACH Registry Investigators. Pulse pressure and risk for cardiovascular events in patients with atherothrombosis: from the REACH registry. J Am Coll Cardiol. 2016;67(4):392-403. [PubMed: 26821627]

43. Bangalore S, Messerli FH, Franklin SS, et al. Pulse pressure and risk of cardiovascular outcomes in patients with hypertension and coronary artery disease: an INternational VErapamil SRtrandolapril STudy (INVEST) analysis. Eur Heart J. 2009;30(11):1395-1401. [PubMed: 19351690]

44. Morgan TO, Anderson AI, MacInnis RJ. ACE inhibitors, beta-blockers, calcium blockers, and diuretics for the control of systolic hypertension. Am J Hypertens. 2001;14(3):241-247. [PubMed: 11281235]

45. Messerli FH, Grossman E, Goldbourt U. Are $\beta$-blockers efficacious as first-line therapy for hypertension in the elderly? JAMA. 1998;279:1903-1907. [PubMed: 9634263]

46. Dahlöf B, Devereux RB, Kjeldsen SE, et al. Cardiovascular morbidity and mortality in the Losartan Intervention For Endpoint Reduction in Hypertension study (LIFE): a randomised trial against atenolol. Lancet. 2002;359(9311):995-1003. [PubMed: 11937178]

47. Wiysonge CS, Bradley HA, Volmink J, et al. Beta-blockers for hypertension. Cochrane Database Syst Rev. 2012;11.

48. Casey DP, Curry TB, Joyner MJ, et al. Acute $\beta$-adrenergic blockade increases aortic wave reflection in young men and women: differing mechanisms between sexes. Hypertension. 2012;59(1):145-150. [PubMed: 22106401]

49. Peart S, Greenberg G. MRC trial of treatment of mild hypertension. BMJ (Clinical research ed.). 1985;291(6491):347. [PubMed: 3926189]

50. ALLHAT Collaborative Research Group. Major outcomes in high-risk hypertensive patients randomized to angiotensin-converting enzyme inhibitor or calcium channel blocker vs diuretic: the Antihypertensive and Lipid-Lowering Treatment to Prevent Heart Attack Trial (ALLHAT). JAMA. 2002;288(23):2981-2997. [PubMed: 12479763] 
51. Pfeffer MA, Braunwald E, Moyé LA, et al. Effect of captopril on mortality and morbidity in patients with left ventricular dysfunction after myocardial infarction: results of the Survival and Ventricular Enlargement Trial. New Engl J Med. 1992;327(10):669-677. [PubMed: 1386652]

52. European Trial on Reduction of Cardiac Events with Perindopril in Stable Coronary Artery Disease Investigators. Efficacy of perindopril in reduction of cardiovascular events among patients with stable coronary artery disease: randomised, double-blind, placebo-controlled, multicentre trial (the EUROPA study). Lancet. 2003;362(9386):782-788. [PubMed: 13678872]

53. Heart Outcomes Prevention Evaluation Study Investigators. Vitamin E supplementation and cardiovascular events in high-risk patients. N Engl J Med. 2000;2000(342):154-160.

54. PEACE Trial Investigators. Angiotensin-converting-enzyme inhibition in stable coronary artery disease. N Engl j Med. 2004;2004(351):2058-2068.

55. Pfeffer MA, McMurray JJ, Velazquez EJ, et al. Valsartan, captopril, or both in myocardial infarction complicated by heart failure, left ventricular dysfunction, or both. N Engl J Med. 2003;349(20):1893-1906. [PubMed: 14610160]

56. Mann JF, Schmieder RE, McQueen M, et al. Renal outcomes with telmisartan, ramipril, or both, in people at high vascular risk (the ONTARGET study): a multicentre, randomised, double-blind, controlled trial. Lancet. 2008;372(9638):547-553. [PubMed: 18707986]

57. Julius S, Kjeldsen SE, Weber M, et al. Outcomes in hypertensive patients at high cardiovascular risk treated with regimens based on valsartan or amlodipine: the VALUE randomised trial. Lancet. 2004;363(9426):2022-2031. [PubMed: 15207952]

58. Mancia G, Fagard R, Narkiewicz K, et al. 2013 ESH/ESC Guidelines for the Management of Arterial Hypertension: the Task Force for the Management of Arterial Hypertension of the European Society of Hypertension (ESH) and of the European Society of Cardiology (ESC). Blood Press. 2013;22(4):193-278. [PubMed: 23777479]

59. James PA, Oparil S, Carter BL, et al. 2014 Evidence-Based Guideline for the Management of High Blood Pressure in Adults: Report from the panel members appointed to the Eighth Joint National Committee (JNC 8). JAMA. 2014;311(5):507-520. [PubMed: 24352797]

60. Williams B, Mancia G, Spiering W, Agabiti Rosei E, Azizi M, Burnier M, Clement DL, Coca A, de Simone G, Dominiczak A, Kahan T, Mahfoud F, Redon J, Ruilope L, Zanchetti A, Kerins M, Kjeldsen SE, Kreutz R, Laurent S, Lip GYH, McManus R, Narkiewicz K, Ruschitzka F, Schmieder RE, Shlyakhto E, Tsioufis C, Aboyans V, Desormais I; ESC Scientific Document Group. 2018 ESC/ESH Guidelines for the management of arterial hypertension. Eur. Heart J. 2018;39(33):3021-3104. [PubMed: 30165516]

61. Kjeldsen SE, Lund-Johansen P, Nilsson PM, et al. Unattended blood pressure measurements in the Systolic Blood Pressure Intervention trial. Hypertension. 2016;67(5):808-812. [PubMed: 27001295]

62. Wright JS, Wall HK, Ritchey MD. Million Hearts 2022: small steps are needed for cardiovascular disease prevention. JAMA. 20189 6. doi:10.1001/jama.2018.13326. [Epub ahead of print]

Expert Rev Cardiovasc Ther. Author manuscript; available in PMC 2019 November 01. 


\section{Key issues}

- $\quad$ Patients with the dyad of hypertension and CAD undergo physiological changes such as endothelial dysfunction, microvascular dysfunction and remodeling. They also experience changes in the coronary auto-regulatory mechanisms as well as in the myocardial structure.

- Due to hindrance in the auto-regulatory mechanisms and concomitant cerebral atherosclerosis, multitude of trials over the last two decades have debated on optimal BP target in these patients and regarding the existence of the J-curve phenomenon.

- $\quad$ Data regarding the optimum SBP and DBP in this population has been mainly driven from observational studies and post hoc analyses of randomized controlled trials as the BP control trials did not focus on this population.

- $\quad$ Patients with the dyad of hypertension and CAD should be treated with antihypertensive agents in line with other risk factors or comorbid conditions. Data regarding the optimum therapeutic goal BP in this population are driven from observational analyses, as well as post-hoc analyses of randomized controlled trials.

- $\quad$ The suggested targeted BP should be $<130 / 80 \mathrm{mmHg}$ while paying particular attention to avoid lowering DBP if SBP $<120 \mathrm{mmHg}$. However, future prospective randomized controlled trials are necessary in this field to further ascertain the optimum therapeutic target in this population. 


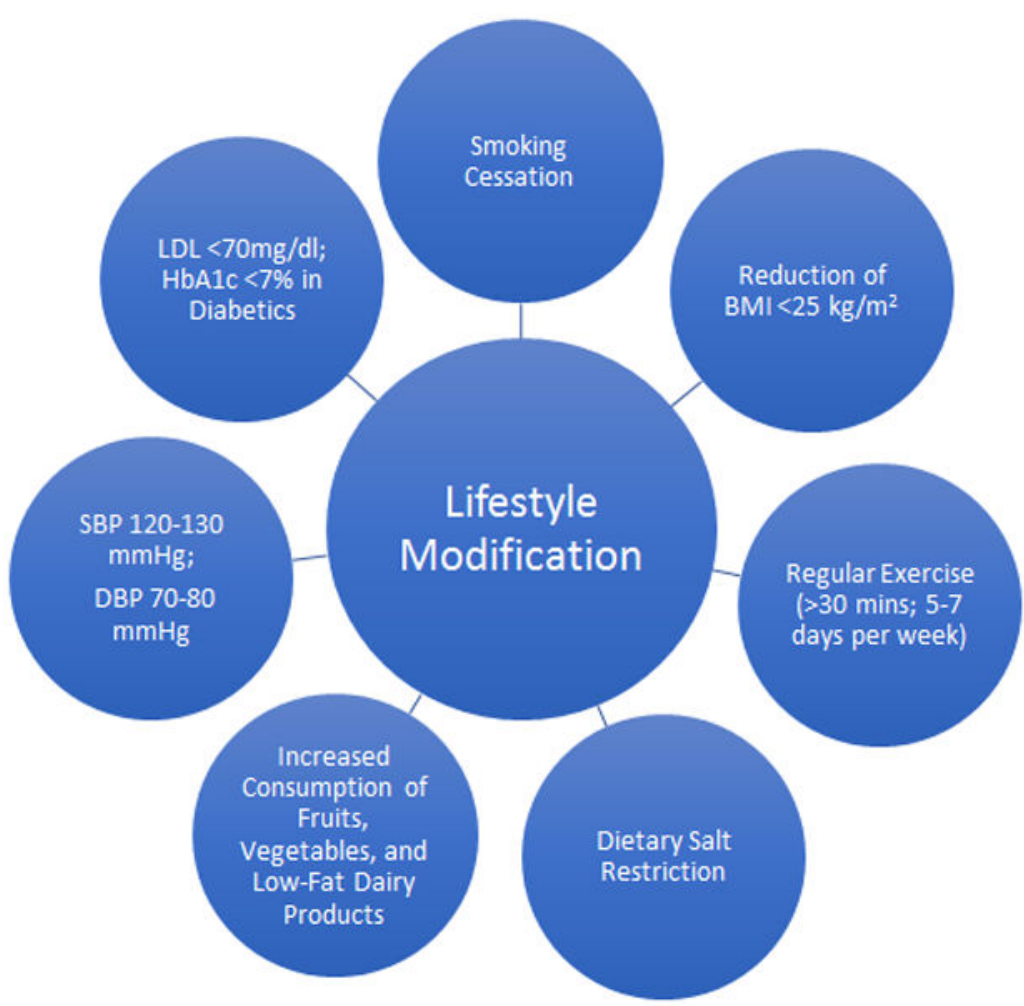

Figure 1.

Schematic diagram of lifestyle modifications for secondary prevention of adverse cardiovascular events in patients with hypertension and coronary artery disease.

$\mathrm{BMI}=$ body mass index; $\mathrm{DBP}=$ diastolic blood pressure HbA1c $=$ hemoglobin A1c; $\mathrm{LDL}=$ low-density lipoprotein; SBP = systolic blood pressure. 


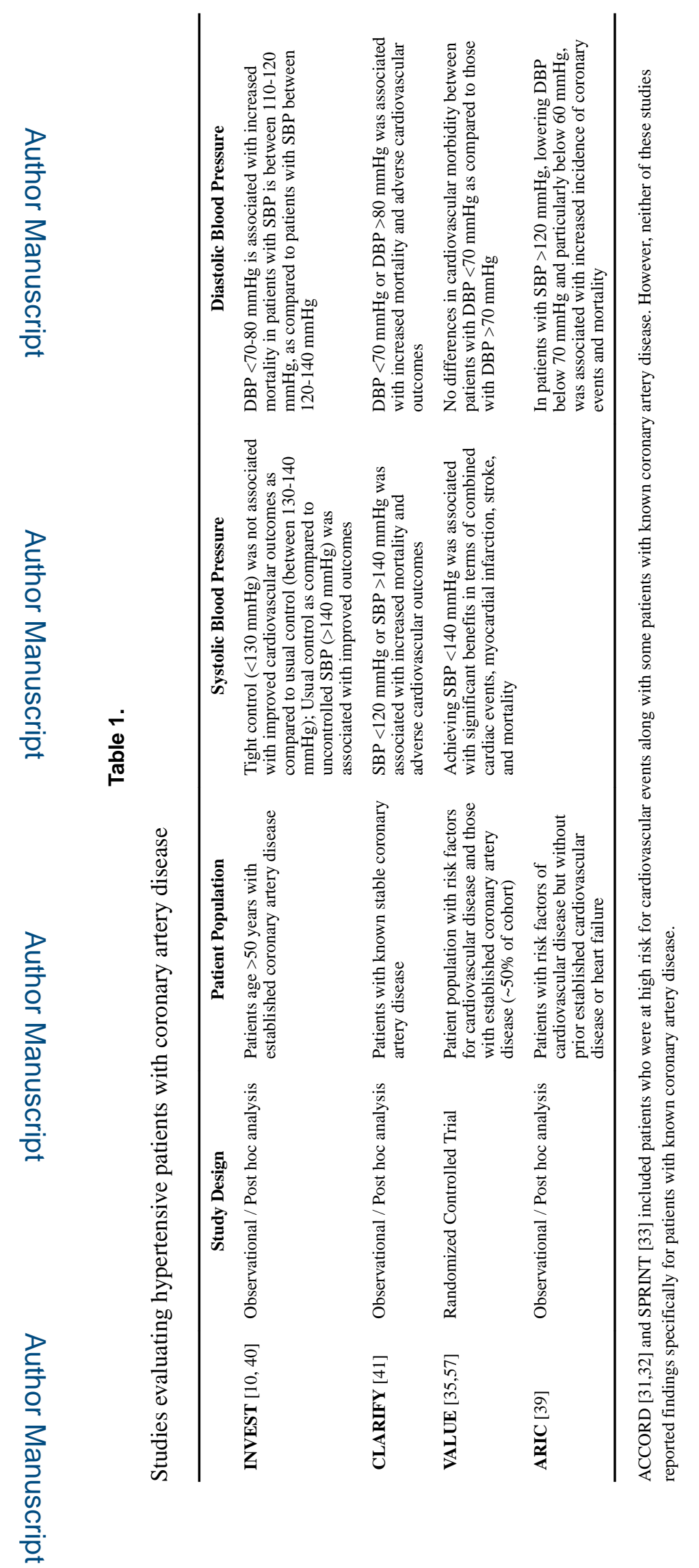

Expert Rev Cardiovasc Ther. Author manuscript; available in PMC 2019 November 01. 


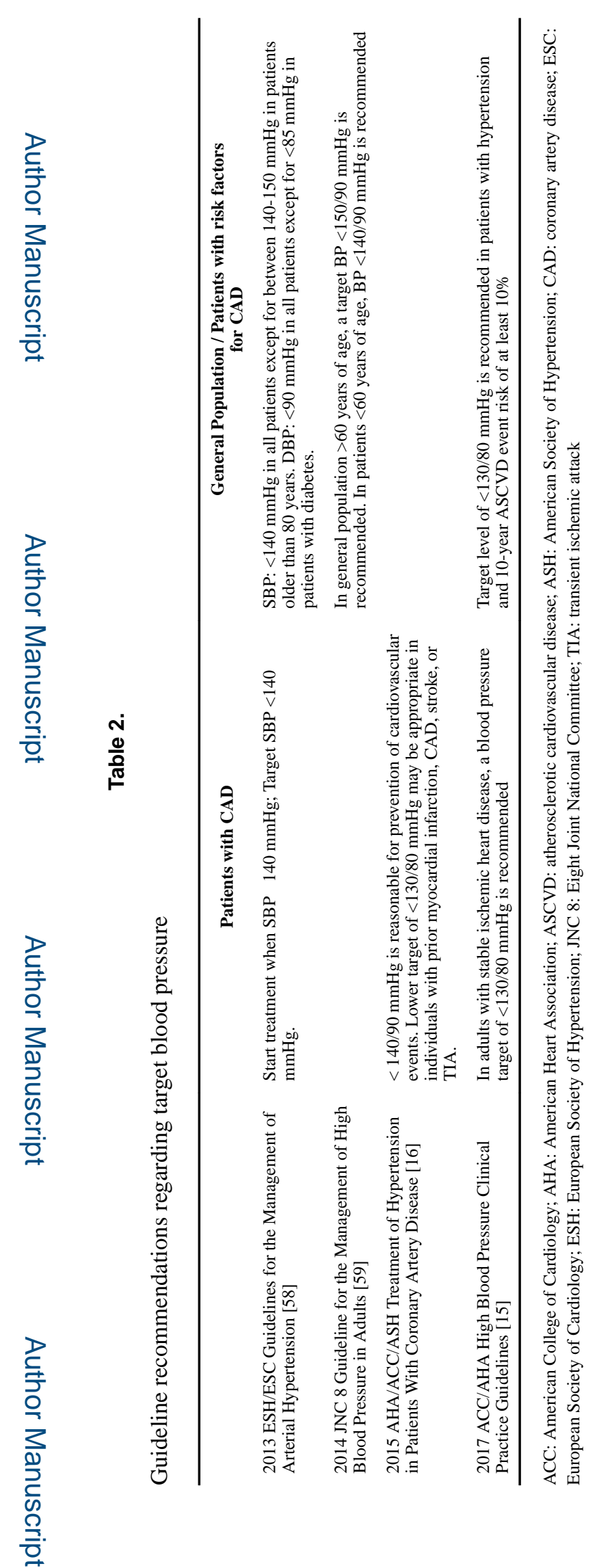

Expert Rev Cardiovasc Ther: Author manuscript; available in PMC 2019 November 01. 